\title{
Comments on the amplitude-phase relationship of asteroid lightcurves
}

\section{Effects of topography, surface scattering properties, and obliquity}

\author{
P. J. Gutiérrez ${ }^{1}$, B. J. R. Davidsson ${ }^{2}$, J. L. Ortiz ${ }^{1}$, R. Rodrigo ${ }^{1}$, and M. J. Vidal-Nuñez ${ }^{1}$ \\ 1 Instituto de Astrofísica de Andalucía-CSIC, PO Box 3004, 18080 Granada, Spain \\ e-mail: pedroj@iaa.es \\ 2 Department of Astronomy and Space Physics, Uppsala University, Box 515, 75120 Uppsala, Sweden
}

Received 11 January 2006 / Accepted 6 March 2006

\section{ABSTRACT}

\begin{abstract}
Aims. We present a theoretical study on the amplitude-phase relationship (APR) for lightcurves of simulated asteroids. Methods. In support of the Rosetta (ESA) mission, we developed a numerical model for the investigation of the light reflectance properties of asteroidal bodies. The code is able to deal with irregular and chemically inhomogeneous surfaces, taking shadowing effects into account. Several standard scattering models have been implemented, which govern local reflectance properties, e.g. the Hapke model and the Lumme-Bowell model. From a kinematic standpoint, the body can move in an arbitrary orbit, and it may rotate in either pure or complex mode with an arbitrary orientation of its angular momentum. As an application of the code, we studied the dependence of the APR on several factors, such as the illumination and observational geometries, overall shape, and large-scale topography, as well as the surface characteristics represented by the parameters in the Hapke and Lumme-Bowell models.

Results. In our study, we find that mineralogy, regolith properties, and small-scale surface roughness (i.e., characteristics embodied in the considered surface scattering models), have a negligible effect on the APR. Furthermore, large-scale topography introduces a rather significant dispersion in the APR slope, on the order of $0.010 \mathrm{mag} \mathrm{deg}^{-1}$. Our simulations suggest that obliquity is the major agent for shaping the APR, causing a $0.020 \mathrm{mag} \mathrm{deg}^{-1}$ dispersion in the APR slope; the larger the obliquity, the smaller the slope of the APR. For intermediate aspect angles, large obliquities could even lead to an amplitude that decreases with the phase angle.
\end{abstract}

Key words. minor planets, asteroids - methods: numerical - solar system: general

\section{Introduction}

Asteroid research is one of the main topics in Solar System science. So far, photometric observations have been the main source of asteroidal data. The lightcurve of an asteroid, i.e. the variation of its magnitude with time, depends strongly on geometric factors, including illumination and viewing geometries and asteroidal shape, as well as factors related to the chemical and physical characteristics of the surface.

Over the years, asteroids have been selected as main or secondary targets of several space missions, such as Galileo (NASA), NEAR Shoemaker (NASA), Hayabusa (Muses-C) (JAXA), and Rosetta (ESA). Sophisticated photometric models including characteristics such as non-spheroidal shape and realistic scattering properties are needed to analyze photometric data collected by such spacecraft.

With this main goal in mind, we developed a numerical code to calculate the synthetic reflectance spectra and related photometric quantities of an asteroidal body. The code is able to deal with fully irregular surfaces, taking shadowing effects into account. At present, the most common light scattering models, such as those of Lumme-Bowell, Hapke, Lommel-Seeliger, Lambert, etc., have been implemented, and the corresponding model parameters are allowed to vary across the surface (i.e., the model objects can be chemically and physically heterogeneous). From a kinematic point of view, the body can have an arbitrary orbit and may rotate either in pure or in complex mode with an arbitrary orientation of its angular momentum. The code has primarily been developed to analyze images of Asteroids 21 Lutetia and 2867 Steins, to be delivered by the OSIRIS instrument onboard Rosetta, but the range of possible applications is of course much wider.

Here, we perform a study on the lightcurve amplitude $A$ of simulated asteroids and its dependence on several factors, such as illumination and observational geometries, the shape of the body, and surface characteristics represented by the parameters involved in different scattering models. The Hapke and the Lumme-Bowell models are considered, and monochromatic irradiation is used for simplicity. The model bodies are assumed to rotate in a pure mode.

To date, several authors have performed studies on the lightcurve amplitude, e.g. Barucci \& Fulchinogni (1982), Karttunen \& Bowell (1989), and Zappalà et al. (1990). These studies are summarized in the next section. To improve upon these previous studies, we considered realistic asteroidal shapes, represented by the Gaussian Random Spheres (Peltoniemi et al. 1989; Muinonen 1996). In addition, the difference between using two complex scattering models is studied.

\section{Previous work}

As stated above, the lightcurve of an asteroid strongly depends on the geometrical circumstances during the observation. The position of an asteroid in space with respect to the Sun and an 
observer, and the instantaneous body orientation, are often described in terms of the four following angles (see, e.g. Barucci $\&$ Fulchinogni 1982): the phase angle $\alpha$, the aspect angle $\beta$, the obliquity $\gamma$, and the rotation angle.

The phase angle is the angular distance between the Sun and the observer, as seen from the asteroid, and depends solely on orbit characteristics. The aspect is the angle between the rotation axis and the line of sight. The obliquity is the angle between a plane $X$ (containing the observer and the spin vector) and the normal to a plane $Y$ (containing the asteroid, the observer, and the Sun). The aspect and obliquity angles therefore specify the orientation of the rotation axis in space. Finally, the rotation angle determines the instantaneous rotational phase of the body. With this parameterization, it is implicitly assumed that the asteroid is rotating around an axis of inertia, generally taken to be the shortest principal axis.

For elongated objects, it is evident that the aspect angle (along with the detailed body shape) has a major influence on the appearance of the lightcurve. Actually, some methods used to determine pole orientations and asteroid shapes, such as the amplitude-aspect method (see e.g. Pospieszalska-Surdej \& Surdej 1985; Magnusson 1986) take advantage of this direct dependence. However, the discovery by Gehrels (1956), that the lightcurve amplitude of Asteroid 20 Massalia increases with phase angle (a behavior that subsequently has been observed for many other asteroids), is a more delicate problem, which lacks a simple explanation.

In the past, several authors have investigated the effect of the angular parameters and shape (represented by ellipsoids with different axis ratios) on the lightcurve amplitude by using theoretical approximations and laboratory models. Here, a number of such investigations are summarized.

For example, Barucci \& Fulchignoni (1982), assuming geometrical scattering ${ }^{1}$ and ellipsoidal body shapes, derived an analytical expression for the lightcurve amplitude as a function of the previously mentioned angles and of the semi-axes of the ellipsoid. An important result of this investigation was that the obliquity has a negligible effect on the lightcurve amplitude for phase angles $\alpha \lesssim 30^{\circ}$ (becoming dominant for larger phase angles). Since most of the asteroids do not reach phase angles larger than $\alpha \approx 30^{\circ}$, it has been widely assumed that the obliquity is unimportant for the lightcurve characteristics.

More recently, Zappalà et al. (1990) performed an extensive study of how the lightcurve amplitude depends on the phase angle and coined the phrase amplitude-phase relationship (APR). The authors first made a theoretical study of the APRs for a set of ellipsoids, assuming geometric scattering. They found that the lightcurve amplitude depends linearly on the phase angle, at least when $\alpha \lesssim 40^{\circ}$. That is, the APR can be written in the form

$A(\alpha)=A\left(0^{\circ}\right)+s(\mathrm{APR}) \cdot \alpha$,

where $A\left(0^{\circ}\right)$ is the lightcurve amplitude at zero phase and $s(\mathrm{APR})$ is the slope of the APR.

Furthermore, the authors studied the lightcurves of a set of laboratory models (ellipsoidal bodies covered by a mixture of plaster of Paris and black carbon were used) in the range $4^{\circ} \leq$ $\alpha \leq 24^{\circ}$, and found that Eq. (1) is also sufficiently accurate in these cases. Considering a larger number of parameter pairs $\left\{A\left(0^{\circ}\right), s(\mathrm{APR})\right\}$, obtained by fitting Eq. (1) to data from numerical and laboratory models (using a variety of ellipsoidal body

1 For geometrical scattering, the observed intensity is proportional to the projected illuminated area, as seen by the observer.

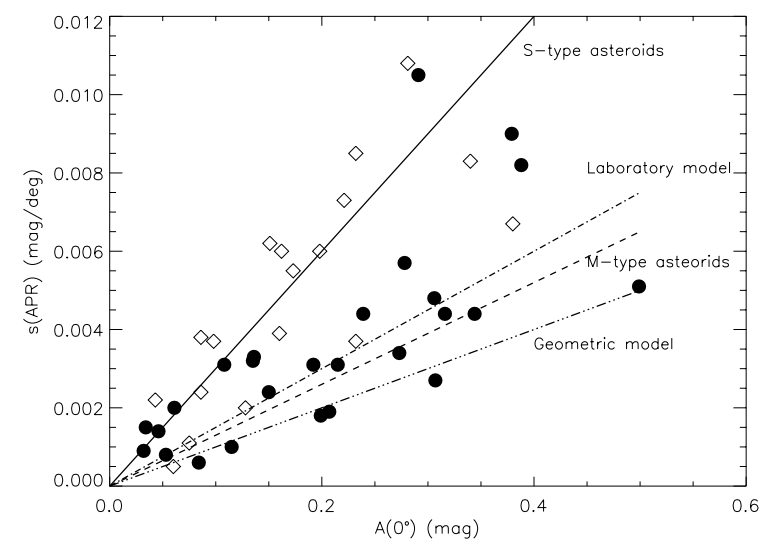

Fig. 1. A reproduction of Fig. 7 from Zappalà et al. (1990). For each considered asteroid, the lightcurve amplitude $A$ versus phase angle $\alpha$ is observed during a particular opposition. A linear least-squares fit to the resulting amplitude-phase relationship (APR) yields a slope, $s(\mathrm{APR})$, plotted here against the amplitude corresponding to zero phase, $A\left(0^{\circ}\right)$, for the linear fit in question. The acquisition of such $\left\{A\left(0^{\circ}\right), s(\mathrm{APR})\right\}$ pairs during several oppositions indicates that $s(\mathrm{APR})$ grows with $A\left(0^{\circ}\right)$, for a particular asteroid. According to Zappalà et al. (1990), this rate of growth is steeper for S-type asteroids, represented by diamonds $(\diamond)$, than for C- or M-asteroids, represented by filled circles $(\bullet)$. The plot also shows such average linear trends for different taxonomic types, laboratory material, and model bodies, as indicated in the figure.

shapes and aspect angles), the authors found a linear relationship between the two, i.e.,

$s(\mathrm{APR})=m \cdot A\left(0^{\circ}\right)$,

where $m$ is a slope parameter.

Substitution of Eq. (2) into Eq. (1) yields a simple and useful relationship, which can be used to reduce an observed amplitude at an arbitrary phase angle $\alpha \lesssim 40^{\circ}$ to zero phase angle.

$A(\alpha)=(1+m \cdot \alpha) A\left(0^{\circ}\right)$.

Zappalà et al. (1990) found that $m=0.010 \mathrm{mag} \mathrm{deg}^{-1}$ for the set of numerical models (i.e., geometrically scattering ellipsoids). For the set of laboratory models, the authors found a slightly steeper slope, $m=0.015 \mathrm{mag} \mathrm{deg}^{-1}$. It is necessary to note that in this study, the authors do not mention which obliquity they used in the calculations and experiments.

Zappalà et al. (1990) concluded their study by analyzing the lightcurves of 33 asteroids belonging to different taxonomic types (S, M, and C). They found that the asteroids in their sample behaved in a similar way, qualitatively speaking, to the geometric and laboratory models, i.e., the lightcurve amplitude depends linearly on the phase angle, and the slope of this linear relationship, $s$ (APR), seems to depend linearly on the amplitude at zero phase angle, $A\left(0^{\circ}\right)$. As an important result, the authors found that the slope $m$ depends on the taxonomic type. For Mand C-type asteroids, they obtained $m=0.013 \mathrm{mag} \mathrm{deg}^{-1}$ and $m=0.015 \mathrm{mag} \mathrm{deg}^{-1}$, respectively, which closely match the values found for the numerical and laboratory models. However, S-type asteroids generally displayed much steeper slopes, $m=$ $0.030 \mathrm{mag} \mathrm{deg}^{-1}$. The authors attributed the variation in $m$ to differences in surface properties and light-scattering characteristics among the considered taxonomic types.

In Fig. 1, we reproduce Fig. 7 of Zappalà et al. (1990), where the authors showed the linear relationship between $s$ (APR) and $A\left(0^{\circ}\right)$ for actual asteroids. In this plot, we have included several lines showing the linear trends found by 


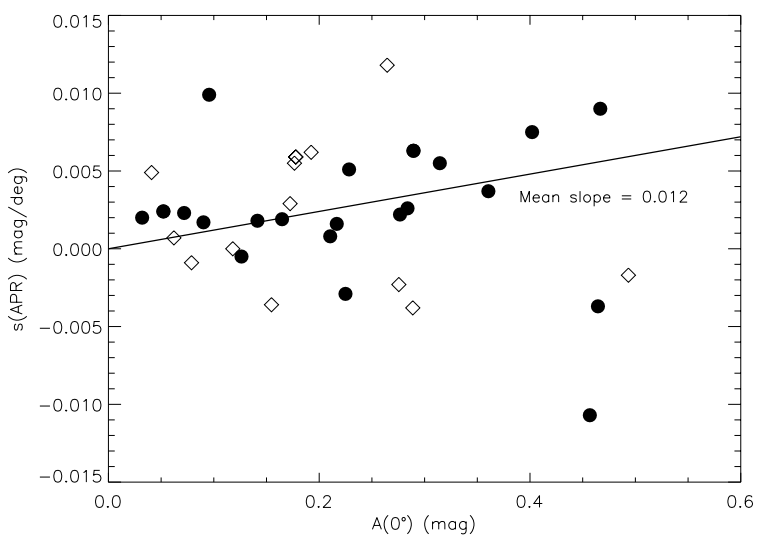

Fig. 2. The slope $s(\mathrm{APR})$ versus the amplitude reduced to zero phase $A\left(0^{\circ}\right)$, as derived by Kristensen \& Dohrup (1997), using the same observational data as in Fig. 1. The solid line is a linear fit to the individual data points and yields the slope $m=0.012 \mathrm{mag} \mathrm{deg}^{-1}$ in Eq. (2) (according to Kristensen \& Dohrup 1997). The symbols are explained in the caption of Fig. 1. According to Kristensen \& Dohrup (1997), the parameter $m$ does not depend on taxonomic type.

Zappalà et al. (1990) for different taxonomic types, numerical models, and laboratory experiments.

To briefly summarize the study performed by Zappalà et al. (1990), it was found that asteroidal lightcurve amplitudes depend approximately linearly on the phase angle, and in addition, the slope of this linear relationship depends linearly on $A\left(0^{\circ}\right)$, at least in first approximation. This finding, represented by Eq. (3), has been widely used to reduce lightcurve amplitudes to a common phase angle, e.g. $\alpha=0^{\circ}$.

It is important to mention that the study of how lightcurve amplitudes change with phase angle for real asteroids is a very complex problem, and to obtain accurate estimates of $m$ is therefore non-trivial. It is necessary to have access to observed lightcurves with high temporal resolution and small observational errors, the phase angle coverage for a particular opposition must be sufficiently large, and such data must be available for several oppositions. In their analysis, Zappalà et al. (1990) therefore had to introduce a subjective weighting system to take the variation in data quality into account.

The difficulty of this work was manifested by Kristensen \& Dohrup (1997) who, by using exactly the same lightcurves as Zappalà et al. (1990), obtained rather different results, especially for S-type asteroids. In particular, Kristensen \& Dohrup (1997) found no evidence for a dependence of $m$ on taxonomic type.

In Fig. 2, we show $s(\mathrm{APR})$ versus $A\left(0^{\circ}\right)$, as obtained by Kristensen \& Dohrup (1997) for the same lightcurve data used by Zappalà et al. (1990). The clear distinction between S-type and C- or M-type asteroids seen in Fig. 1 is no longer present. Kristensen \& Dohrup (1997) even found cases where the lightcurve amplitude decreased with the phase angle, i.e., $s(\mathrm{APR})<0$. For their complete sample, Kristensen \& Dohrup (1997) estimated $m=0.0120 \pm 0.0046 \mathrm{mag} \mathrm{deg}^{-1}$, a value that conforms with the numerical calculations and laboratory experiments performed by Zappalà et al. (1990).

Another theoretical study of the APR was performed by Karttunen \& Bowell (1989). By using different ellipsoidal body shapes and applying the Lumme-Bowell scattering law, these authors found non-linear APRs, especially in the range $0^{\circ} \lesssim \alpha \lesssim 5^{\circ}$ (i.e., close to opposition). Zappalà et al. (1990) commented on the discrepancy resulting from the usage of different scattering laws and noted that the lack of observational data at very small phase angles and the uncertainties in measured lightcurve amplitudes made it impossible to favor one of the two approaches.

As for the orientation parameters, Karttunen \& Bowell (1989) only considered two extreme aspect angles $\left(\beta=0^{\circ}\right.$ and $\beta=90^{\circ}$ ), and they did not mention the obliquity used in the calculations (presumably, it was assumed to be zero). On the other hand, Karttunen \& Bowell (1989) studied the dependence of the APR on the Lumme-Bowell scattering parameters and found that the APR was generally insensitive to these parameters. The mineralogy and texture of the surface were therefore considered to have a negligible effect on the APR, implying that the dependence of, e.g., $m$ on the taxonomic type should be small. However, it is important to remember that these results were obtained for a particular body shape, the smooth ellipsoid.

So far, most of the theoretical studies of asteroidal photometric behavior have been performed by using ellipsoids to represent the body shape, and geometric scattering has often been assumed to calculate the amount of reflected light. In principle, it is expected that a non-spheroidal shape and topography could play some role in the photometric behavior of asteroids, therefore affecting the APR. Moreover, based on results from geometric scattering, it is generally assumed that obliquity does not play any role for the APR, at least for relatively small phase angles. To the best of our knowledge, this has not been confirmed for more complex scattering models. To address these problems we study the amplitude-phase relationship for a number of different irregular bodies and combinations of aspect and obliquity angles, using two different scattering laws (Lumme-Bowell and Hapke). Our work can be considered a generalization of the study by Karttunen \& Bowell (1989).

\section{Model, parameters, and method}

In the following, the method for generating irregular model bodies and the scattering laws are described. Furthermore, the considered numerical experiments and the code evaluation procedure are summarized.

\subsection{Shape and photometry}

As for the shape of the model asteroids, 16 different Gaussian random shapes, so-called gaussinos (Peltoniemi et al. 1989; Muinonen 1996) have been considered here. These irregular shapes are based on lognormal statistics, and they are fully described by the mean radius and the covariance function (see, e.g., Muinonen 1996 for details). Muinonen \& Lagerros (1998) demonstrated that these mathematical surfaces can successfully describe the actual shape of several minor bodies of the Solar System. These authors obtained the discrete spectrum of the Legendre coefficients of the covariance function for a set of both "small" and "large" minor bodies (the transition roughly occurs at a mean radius of $20-30 \mathrm{~km}$ ). In the current paper, we used these covariance functions to randomly generate eight small bodies and eight large bodies. Small bodies are comparably irregular, and in the present work, elongations $^{2}$ ranging from $1: 1.15: 1.57$ to $1: 1.24: 2.97$ are considered. Large objects, which are more spherical due to the increasing importance of self-gravity, have elongations ranging from $1: 1.03: 1.15$ to $1: 1.26: 1.6$ (see Table 1 ) here.

\footnotetext{
2 Here, a triaxial ellipsoid is fitted to the body surface with a leastsquares procedure, and the semi-axes of this ellipsoid are taken to represent the overall shape of the object.
} 
Table 1. Characteristics of the irregular bodies used in this study. In the columns labeled "a/c" and "b/c", we show the semi-axis ratios of the ellipsoid best fitting the corresponding irregular body. The column labeled "Deviation" shows the mean deviation (in percent) of the irregular body from the best-fit ellipsoid. This "Deviation" represents the cumulative difference between the local radius of the irregular body and that of the best-fit ellipsoid.

\begin{tabular}{c|ccc|c|ccc}
\hline \hline Body & $\mathrm{a} / \mathrm{c}$ & $\mathrm{b} / \mathrm{c}$ & Deviation (\%) & Body & $\mathrm{a} / \mathrm{c}$ & $\mathrm{b} / \mathrm{c}$ & Deviation (\%) \\
\hline$\# 1$ & 1.94 & 1.35 & 4.5 & $\# 9$ & 1.37 & 1.15 & 6.2 \\
$\# 2$ & 1.57 & 1.15 & 4.6 & $\# 10$ & 1.47 & 1.22 & 2.9 \\
$\# 3$ & 2.97 & 1.24 & 5.9 & $\# 11$ & 1.67 & 1.26 & 3.0 \\
$\# 4$ & 1.42 & 1.23 & 4.1 & $\# 12$ & 1.49 & 1.25 & 2.5 \\
$\# 5$ & 2.46 & 1.30 & 5.1 & $\# 13$ & 1.45 & 1.09 & 3.1 \\
$\# 6$ & 2.55 & 1.39 & 6.6 & $\# 14$ & 1.15 & 1.03 & 2.9 \\
$\# 7$ & 2.27 & 1.26 & 7.0 & $\# 15$ & 1.55 & 1.17 & 2.4 \\
$\# 8$ & 1.81 & 1.27 & 4.7 & $\# 16$ & 1.39 & 1.15 & 2.7 \\
\hline
\end{tabular}
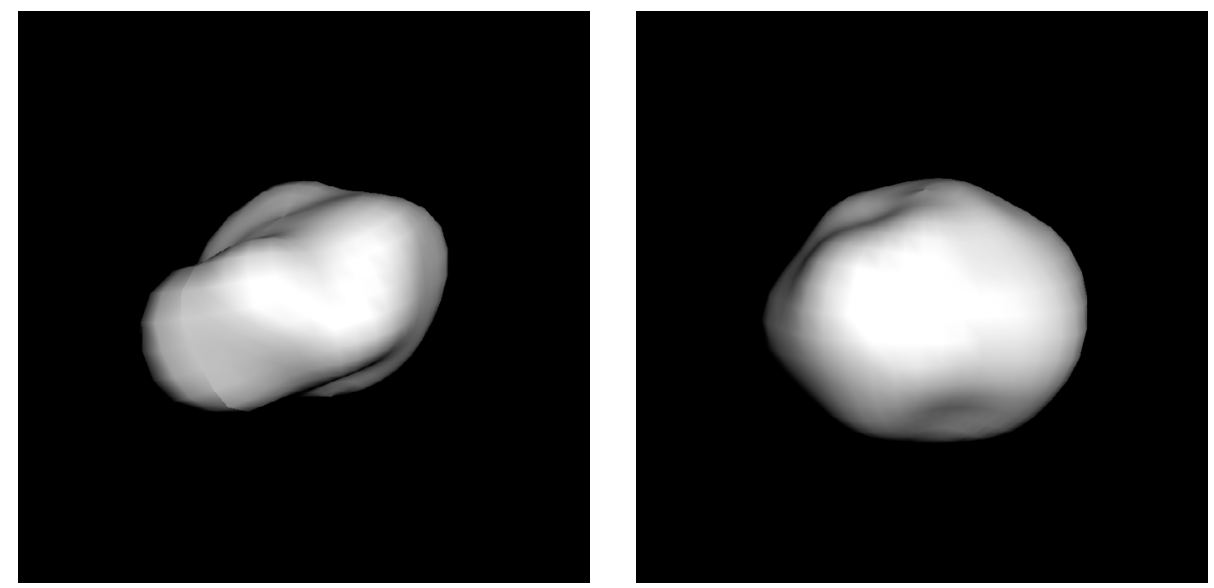

Fig. 3. Examples of gaussinos used in the present study. These shapes have been generated by using the covariance functions estimated by Muinonen \& Lagerros (1998) for "small" bodies (left, object \#1) and for "large" bodies (right, object \#9).

In Fig. 3, two gaussinos used in this study are shown. In this figure, it can be seen that these bodies have some topography that can potentially produce shadowing. In the calculations, shadows are taken into account as described by Gutiérrez et al. (2000).

As for the scattering model, the Lumme-Bowell (Lumme et al. 1980; Lumme \& Bowell 1981a,b) and the Hapke (1981, 1984) theories have been used to calculate the bidirectional reflectance across the surface, which is then integrated to yield the flux seen by the observer. Since both scattering theories are considered standard tools in reflectance spectroscopy applications, we do not describe the main hypothesis or reproduce the essential formulas here, instead we merely define our notation and discuss the range of typical parameter values.

The Lumme-Bowell theory was implemented as described by Karttunen (1989) and by Karttunen \& Bowell (1989). In this version of the theory, the single-particle function is described by the Henyey-Greenstein phase function, and the reflectance depends on four parameters representing different surface properties: the single-scattering albedo $\omega_{0}$, the asymmetry factor $g$, the roughness $\rho$, and the volume density $D$. A fifth parameter, the area covered by holes $q$ is always taken to be unity in this paper, as it was by Karttunen \& Bowell (1989).

In regards to the photometric model by Hapke, we implemented the formalism described by Hapke (1984), including the correction for macroscopic roughness. As before, the singlescattering function is represented by the Henyey-Greenstein phase function. In this formalism, the reflectance depends on five parameters: the single-scattering albedo $\omega_{0}$, the asymmetry factor $g$, the amplitude of the opposition effect $B_{0}$, the backscatter parameter $h$ related to the porosity of the regolith, and the mean slope angle $\theta$ representing the surface roughness.

Both photometric models have been widely used in the literature to interpret lightcurves and phase curves of various atmosphere-less bodies in the Solar System, and standard parameter values have been defined for the taxonomic types of asteroids (see, e.g., Karttunen \& Bowell 1989 for the standard values of the Lumme-Bowell model and Helfenstein \& Ververka 1989 for standard Hapke parameters). These standard values are summarized in Table 2.

\subsection{Code evaluation}

For practical reasons, it is necessary to divide the model body surfaces into a number of locally flat surface facets, which are used to calculate local illumination and reflectance properties. Here, triangular facets with an angular bin size of $9^{\circ}$ were used, and it has been verified that the discretization of the surface does not introduce any artificial effect in the APR. We calculated the size of the illuminated projected cross-sections, as seen by an observer, for a number of observational geometries and ellipsoid body shapes, and compared such numerical results with analytical formulas given by Barucci \& Fulchignoni (1982) and by Pospieszalska-Surdej \& Surdej (1985). We found that with the applied discretization, the agreement is excellent for $\alpha \lesssim 60^{\circ}$. At larger phase angles, the numerical APR drops slightly faster with $\alpha$ than expected, but this behavior is only seen for particular aspect and obliquity angles, and tends to zero as the number of surface facets is increased. Thus, for the conditions under study 
Table 2. Parameter values applied for the photometric models in the current work.

\begin{tabular}{c|ccccc|c|ccccc}
\hline \hline & \multicolumn{4}{|c|}{ Lumme \& Bowell } & \multicolumn{5}{c}{ Hapke } \\
Sim & $\omega_{0}$ & $g$ & $\rho$ & $D$ & $q$ & Sim & $\omega_{0}$ & $g$ & $\theta$ & $h$ & $B_{0}$ \\
\hline C-type & 0.18 & -0.04 & 1.17 & 0.37 & 1.0 & C-type & 0.037 & -0.47 & 20 & 0.025 & 1.03 \\
S-type & 0.54 & -0.07 & 1.17 & 0.37 & 1.0 & S-type & 0.27 & -0.27 & 20 & 0.08 & 1.60 \\
\hline Nominal & 0.35 & -0.055 & 1.17 & 0.37 & 1.0 & Nominal & 0.1 & -0.20 & 20 & 0.05 & 1.30 \\
\#A1 & $\mathbf{0 . 1}$ & -0.055 & 1.17 & 0.37 & 1.0 & \#A2 & $\mathbf{0 . 0 2}$ & -0.20 & 20 & 0.05 & 1.30 \\
\#B1 & $\mathbf{0 . 6}$ & -0.055 & 1.17 & 0.37 & 1.0 & \#B2 & $\mathbf{0 . 4 0}$ & -0.20 & 20 & 0.05 & 1.30 \\
\#C1 & 0.35 & $\mathbf{- 0 . 2 0}$ & 1.17 & 0.37 & 1.0 & \#C2 & 0.1 & $\mathbf{- 0 . 5 0}$ & 20 & 0.05 & 1.30 \\
\#D1 & 0.35 & $\mathbf{+ 0 . 2 0}$ & 1.17 & 0.37 & 1.0 & \#D2 & 0.1 & $\mathbf{+ 0 . 2 0}$ & 20 & 0.05 & 1.30 \\
\#E1 & 0.35 & -0.055 & $\mathbf{0 . 5 0}$ & 0.37 & 1.0 & \#E2 & 0.1 & -0.20 & $\mathbf{1 0}$ & 0.05 & 1.30 \\
\#F1 & 0.35 & -0.055 & $\mathbf{1 . 5 0}$ & 0.37 & 1.0 & \#F2 & 0.1 & -0.20 & $\mathbf{3 5}$ & 0.05 & 1.30 \\
\#G1 & 0.35 & -0.055 & 1.17 & $\mathbf{0 . 2 0}$ & 1.0 & \#G2 & 0.1 & -0.20 & 20 & $\mathbf{0 . 0 1}$ & 1.30 \\
\#H1 & 0.35 & -0.055 & 1.17 & $\mathbf{0 . 8 0}$ & 1.0 & \#H2 & 0.1 & -0.20 & 20 & $\mathbf{0 . 0 9}$ & 1.30 \\
- & - & - & - & - & - & \#I2 & 0.1 & -0.20 & 20 & 0.05 & $\mathbf{1 . 0}$ \\
- & - & - & - & - & - & \#J2 & 0.1 & -0.20 & 20 & 0.05 & $\mathbf{3 . 3}$ \\
\hline
\end{tabular}

in the following, numerical artifacts due to surface discretization can be considered non-existent.

Concerning the photometric models, Lumme \& Bowell (1981b) and Hapke (1984) have (for their respective models) provided analytical expressions for the integrated reflectance expected for a sphere when $\alpha \lesssim 40^{\circ}$. It has been verified that our code reproduces these formulas perfectly.

The considered photometric models allow us to calculate the bidirectional reflectance for locally flat surfaces, i.e., the light intensity at a certain emission angle, compared to the solar illumination at a certain incidence angle. However, for irregular bodies, it is possible that a particular surface facet is illuminated not only by the Sun, but also by reflected light originating from other facets. To evaluate the importance of such self-illumination, we calculated the exact flux reflected by several of the irregular model bodies by solving the corresponding coupled system (assuming a Lambert scattering law). We then compared the exact flux with the solution obtained by taking only two or three consecutive reflections into account. For typical values of the Bond albedo, light reflected twice by the surface contributes approximately $0.01 \%$ of the total flux (i.e., compared to the exact solution). Light reflected three times contributes less than $0.001 \%$ to the total flux. Therefore, self-illumination is considered negligible here, and it is not taken into account in our simulations.

\subsection{Procedure}

We performed two sets of simulations. The first one focuses on the effect of varying the scattering parameters (e.g. investigating the importance of surface composition and texture), and the second one on the effects of geometric factors (in particular, the dependence on obliquity and aspect angles). In both cases, a variety of irregular body shapes are considered.

To study the effect of the scattering parameters, we calculated the lightcurve amplitude for six irregular objects (three small and three large ones) using all the model parameter sets shown in Table 2 (labeled \#A through \#J). To cover the relevant region of parameter space for the Lumme-Bowell model, we use the same ranges considered by Karttunen \& Bowell (1989). In terms of the Hapke model, a compilation of scattering parameters for real objects made by Buratti et al. (2004) are used to define the ranges. The considered fraction of parameter space includes the areas occupied by $\mathrm{C}$ - and S-type asteroids. In Table 2, we also define a nominal reference case for both considered scattering laws, defined by averaging the parameters for C- and S-type asteroids. In the set of simulations targeting the importance of surface scattering properties, aspect and obliquity angles of $0^{\circ}, 45^{\circ}$, and $90^{\circ}$ are considered.

To study the effect of the geometric factors (shape and angular parameters) on the APR, we calculated the lightcurve amplitude for all 16 irregular objects, using the C- and S-type scattering parameters for both the Lumme-Bowell and Hapke models (see Table 2). Calculations were performed with a $5^{\circ}$ resolution in both aspect and obliquity angles, in the range $0^{\circ} \leq$ $\{\beta, \gamma\} \leq 90^{\circ}$. All simulations in the current study were made for $0^{\circ} \leq \alpha \leq 40^{\circ}$, using a $2^{\circ}$ resolution.

\section{Results}

A consequence of the theoretical investigation by Zappalà et al. (1990) is that two smooth, geometrically scattering, ellipsoidal bodies with very different elongations will produce the same APR, if the aspect angles are such that the two bodies have the same lightcurve amplitude at zero phase angle. In other words, given the limitations of the theoretical model, the parameter $m$ is expected to be a constant. To account for the empirical dispersion in $m$ (i.e., the variation in $s(\mathrm{APR})$ for a particular $A\left(0^{\circ}\right)$ ) that is clearly present among real asteroids (see Figs. 1 and 2), it is necessary to find the responsible physical mechanism. In this paper, three different alternatives are studied:

1. Differences in scattering properties (caused by variation in mineralogy and surface texture among asteroids ${ }^{3}$ ).

2. Presence of surface topography, which introduces local shadowing.

3. Importance of obliquity, for small phase angles also (when smooth, geometrically scattering ellipsoidal bodies are replaced with irregular model bodies with realistic scattering properties).

Furthermore, some important differences between the LummeBowell and Hapke scattering models are discussed.

\subsection{Comments on the APR versus scattering parameters}

To investigate the sensitivity of the APR to changes in the scattering parameters, the lightcurve amplitudes resulting from applying the parameter sets \#A through \#J (see Table 2) were

\footnotetext{
${ }^{3}$ Expressions like "texture", "mineralogy", etc. used throughout this paper are meant to correspond to the scattering parameters associated with them.
} 

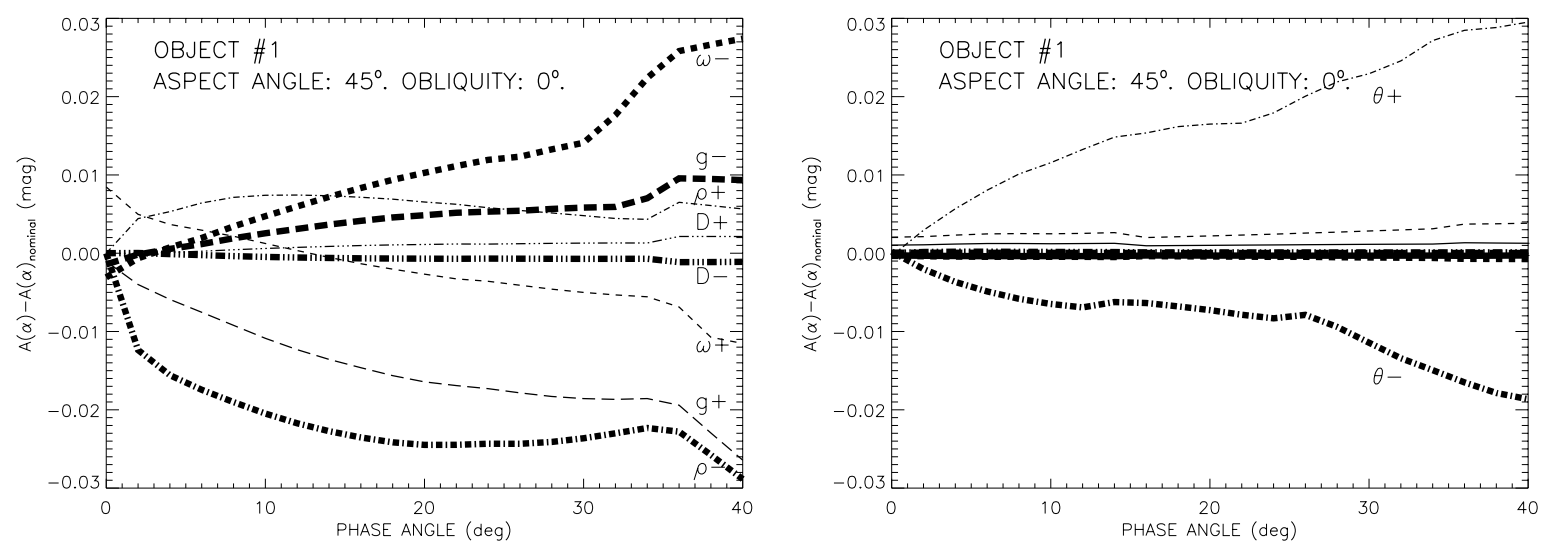

Fig. 4. The difference between lightcurve amplitudes obtained for various scattering parameter sets (\#A through \#J, as specified in Table 2), with respect to the lightcurve amplitude for a nominal scattering parameter set, i.e., $A-A_{\text {nominal }}$ versus phase angle $\alpha$. The left and right panels show results obtained for the Lumme-Bowell and Hapke scattering laws, respectively. Each curve is labeled with the parameter that is currently deviating with respect to the nominal parameter set (the symbols "-" and "+" indicate a relative decrease or increase in the parameter value with respect to the nominal case, respectively). Note that only the surface roughness parameter $\theta$ results in a visible modification of the APR in the right panel, which is why the remaining curves are not labeled. The considered aspect and obliquity angles are indicated in the figure.

compared to the amplitude obtained for the nominal parameter set, i.e., $A(\alpha)-A(\alpha)_{\text {nominal }}$. Figure 4 shows an example of $A(\alpha)-A(\alpha)$ nominal obtained for a particular body shape, aspect angle, and obliquity angle, which is representative of the entire set of considered models. The left panel shows results obtained for the Lumme-Bowell scattering law, while the corresponding results for the Hapke model are shown in the right panel.

Generally, the quantity $\mid A-A_{\text {nominal }}$ is virtually zero at $\alpha=0^{\circ}$, but grows with the phase angle, which means that $A\left(0^{\circ}\right)$ appears to be rather insensitive to surface composition and texture properties, while the slope $s(\mathrm{APR})$ is more dependent on the surface characteristics.

Results obtained for the Lumme-Bowell scattering indicate that the APR has a tendency of becoming steeper (i.e., $s$ (APR) increases) as $\omega_{0}$ or $g$ decrease and $\rho$ increases. In other words, a surface that is dark, back-scattering, and rough would tend to produce a more rapid increase in the lightcurve amplitude with the phase angle, than a brighter, isotropically (or forward) scattering, and less rough surface. The porosity of the surface material seems to have a comparatively small effect on $s(\mathrm{APR})$.

As for Hapke scattering, the considered variation in $\omega_{0}, g$, $h$, and $B_{0}$ hardly leads to any change in the lightcurve amplitude, with respect to the nominal case. The only parameter with a more substantial influence is $\theta$, causing an increase in $s$ (APR) as it grows. In other words, Hapke theory predicts that a rougher surface tends to yield a steeper enhancement in $A$ with an increasing phase angle, than a smoother surface (which is qualitatively consistent with the Lumme-Bowell theory). However, the Hapke theory does not foresee an equally strong dependence of $s$ (APR) on the surface albedo and phase function of the surface particles, as does the Lumme-Bowell theory.

Nevertheless, it is necessary to point out that the rather large variations in the photometric parameters considered here only yield changes in the lightcurve amplitude on the order of $0.01 \mathrm{mag}$ for $\alpha \lesssim 30^{\circ}$, which is similar to the typical observational error. From a practical point of view, it therefore seems that surface mineralogy and texture, and thereby taxonomic type (as represented in the scattering models used in this study), can hardly have an observable effect on the APR of asteroids. This confirms the results already obtained by Karttunen \& Bowell (1989) for the Lumme-Bowell model and extends this statement to include the Hapke model. As a consequence, it currently appears difficult to provide theoretical support for the finding of Zappalà et al. (1990), i.e., that $m$ depends on the taxonomic type. Our results are more in line with the investigation by Kristensen \& Dohrup (1997), who found no systematic difference in the APR for S- and C-type asteroids.

\subsection{Comments on the APR versus topography}

To investigate the importance of topography (or irregular shape, in general), one can study the APRs of various irregular bodies with similar overall shapes and aspect and obliquity angles, and the same surface composition. Among our objects, \#9 and \#16 have very similar overall shape, but very different topography, object \#9 being much more irregular than object \#16 (see Table 1). In Fig. 5, the APRs for these two irregular bodies are shown for an aspect angle of $45^{\circ}$ and an obliquity of $0^{\circ}$. The left and right panels of Fig. 5 show the results for the LummeBowell and Hapke scattering theories, respectively, for C-type scattering parameters according to Table 2.

From Fig. 5, it is immediately evident that topography can indeed have a significant effect on the APR. For the Lumme \& Bowell model, objects \#9 and \#16 (having similar overall shape) show different $A\left(0^{\circ}\right)$, being larger for object \#9. Nevertheless, the slope of the APR corresponding to object \#16 is larger than that of object \#9. This would indicate that, for these cases, topography produces a diminishing slope as $A\left(0^{\circ}\right)$ increases, a behavior differing from the predicted one by Zappalà et al. (1990). The Hapke model (Fig. 5, plot on the right) for these objects yields a different effect. For this scattering model, objects \#9 and \#16 show very similar slopes of the APR, but a different $A\left(0^{\circ}\right)$. This would necessarily introduce some dispersion in the $\left\{A\left(0^{\circ}\right), s(\mathrm{APR})\right\}$ relationship (instead of being linear).

In Fig. 5, the APRs for three additional objects (\#2, \#3, and \#8) are also shown. These objects have different overall shapes but, by coincidence, display similar $A\left(0^{\circ}\right)$ for an aspect angle of $45^{\circ}$. It can be seen that the difference in lightcurve amplitude among the bodies reaches $\sim 0.1 \mathrm{mag}$ around $\alpha \approx 30^{\circ}$, which is substantial in comparison with the amplitudes themselves. A dispersion in $m$ caused by topography/shape is therefore expected, since the APR slopes $s($ APR) are different for bodies with the same $A\left(0^{\circ}\right)$. 

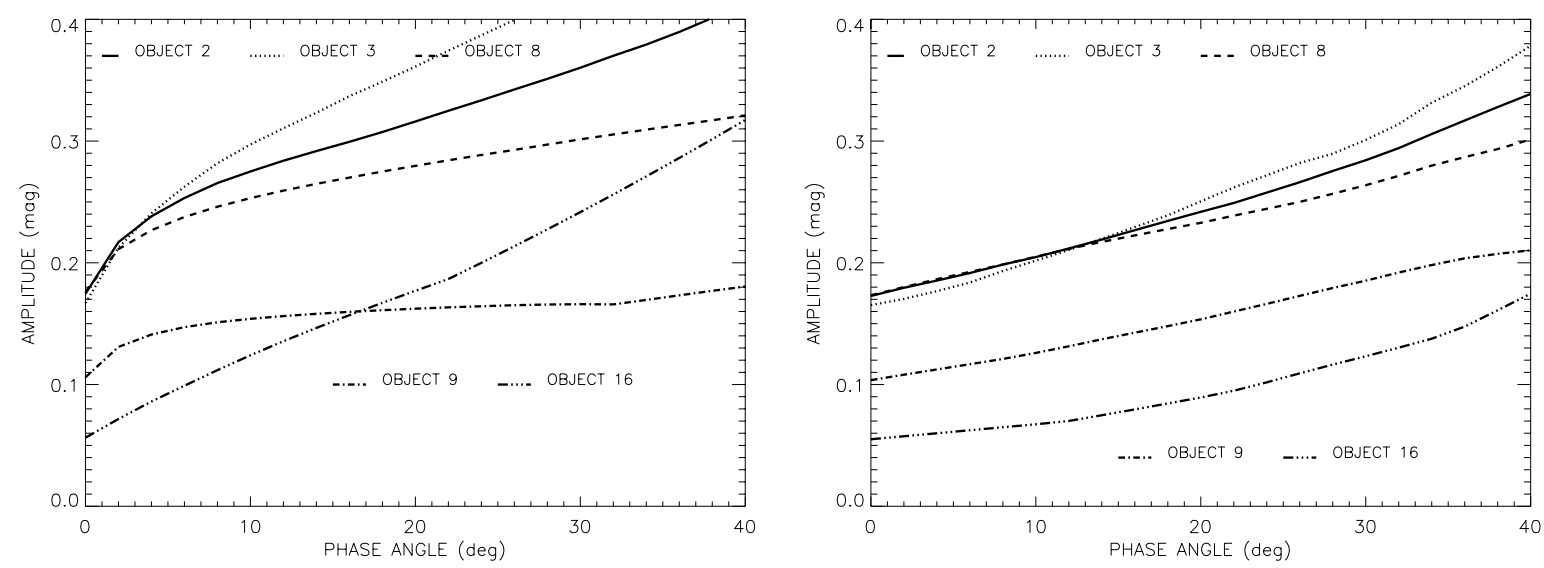

Fig. 5. The lightcurve amplitude $A$ versus phase angle $\alpha$ (i.e., amplitude-phase relationships, APRs) for different irregular bodies considered at an aspect angle $\beta=45^{\circ}$. The obliquity is $\gamma=0^{\circ}$ for all considered cases. All bodies are characterized by scattering parameters corresponding to C-type asteroids (see Table 2). Results for the Lumme-Bowell and Hapke scattering laws are shown in the left and right panels, respectively. Detailed information about the shapes for the considered objects can be found in Table 1 .

We have calculated $m$ values according to Eq. (2) for all 16 body shapes (estimating $A\left(0^{\circ}\right)$ and $s(\mathrm{APR})$ for 19 aspect angles, due to the $5^{\circ}$ resolution in the interval $0^{\circ} \leq \beta \leq 90^{\circ}$, and consistently using an obliquity $\gamma=0^{\circ}$ ). Remembering the investigation by Zappalà et al. (1990), variations in $m$ due to differences in overall shape (elongation) is not expected per se, as long as the surfaces are smooth. However, the irregular bodies considered here give rise to shadowing phenomena, which are expected to have a significant effect on the lightcurves. In fact, the irregular bodies resulted in a median slope ${ }^{4}$ of $m=0.028 \pm 0.007 \mathrm{mag} \mathrm{deg}^{-1}$ for Lumme-Bowell scattering, and $m=0.025 \pm 0.008 \mathrm{mag} \mathrm{deg}^{-1}$ for Hapke scattering, with errors representing $1-\sigma$ of the dispersion in both cases. Thus, according to these simulations, shape and topography would produce a $1-\sigma$ dispersion of about $0.01 \mathrm{mag} \mathrm{deg}^{-1}$ in the $\left\{A\left(0^{\circ}\right), s(\mathrm{APR})\right\}$ relationship.

It is interesting to study whether the degree of irregularity (i.e., the deviation from a smooth surface) has a systematic influence on $m$. We therefore compare the APRs for model objects obtained by applying lognormal statistics for small bodies (expected to be more irregular) with those obtained for large bodies (expected to be more smooth). It is found that, for an obliquity $\gamma=0^{\circ}$, the small bodies in our sample yield the median value $m=0.029 \pm 0.006 \mathrm{mag} \mathrm{deg}^{-1}$, while the large ones yield $m=0.026 \pm 0.007 \mathrm{mag} \mathrm{deg}^{-1}(1-\sigma$ errors$)$, for LummeBowell scattering. The corresponding numbers for Hapke scattering are $m=0.027 \pm 0.007 \mathrm{mag} \mathrm{deg}^{-1}$ (small) and $m=$ $0.023 \pm 0.008 \mathrm{mag} \mathrm{deg}^{-1}$ (large). Hence, there is a weak tendency that more irregular bodies (i.e., the ones displaying a higher degree of shadowing) yield larger $m$-values than smoother bodies. However, the overlap for individual objects is substantial, which means that it seems virtually impossible to say if an individual asteroid is more or less irregular, just based on an empirical $m$ value. In any case, the general conclusion must be that topography and the resulting shadowing effects are important contributors to the empirical dispersion in $m$ values.

It is important to mention that albedo variegation influences the asteroid lightcurve in a manner that is indistinguishable

${ }^{4}$ Median averaging of the 16 slopes for the different objects. For the Lumme-Bowell law, we obtained a slope $m$ ranging from 0.0018 up to $0.037 \mathrm{mag} \mathrm{deg}^{-1}$. For the Hapke model, the slope $m$ obtained in our simulations for the different bodies ranges from 0.016 up to $0.041 \mathrm{mag} \mathrm{deg}^{-1}$. from those of an irregular shape ${ }^{5}$ (Russell 1906). Therefore, it is expected that surface variegation may produce a dispersion in $m$ similar in magnitude to that caused by topography.

Finally, it is noted that the Lumme-Bowell and Hapke scattering models may predict rather different asteroid lightcurves, even when the same body shape and taxonomic type is considered. This is illustrated by Fig. 5, where results for both models can directly be compared. Some differences between the considered scattering models are addressed in Sect. 4.4.

\subsection{Comments on the APR versus obliquity}

As shown by Barucci \& Fulchinogni (1982), geometrical scattering combined with smooth ellipsoidal body shapes leads to a totally negligible importance of the obliquity angle $\gamma$ (for observationally relevant phase angles, i.e., $\beta \lesssim 30^{\circ}$ ). Therefore, subsequent investigations, e.g., by Karttunen \& Bowell (1989), Zappalà et al. (1990), and others, have not dwelt upon this issue. However, when introducing irregular bodies into the analysis, particularly in combination with scattering laws being more realistic than geometric scattering, we were motivated to re investigate the question.

Figure 6 shows APRs calculated for an irregular body for six different combinations of aspect angles $\left(\beta=0^{\circ}, 45^{\circ}\right.$, or $\left.90^{\circ}\right)$ and obliquity angles $\left(\gamma=5^{\circ}\right.$ or $\left.85^{\circ}\right)$. Geometrical scattering (top panel) does not reveal a strong dependence of the APR on $\gamma$ for any of the considered aspect angles, which is in line with previous works. However, Lumme-Bowell scattering (middle panel) and Hapke scattering (bottom panel), show a substantially stronger dependence, in particular for the intermediate aspect angle $\left(\beta=45^{\circ}\right)$. At observationally large phase angles $\left(\alpha \sim 30^{\circ}\right)$, the dispersion in the lightcurve amplitude caused by obliquity can be a few times $0.1 \mathrm{mag}$, which must be considered substantial. Furthermore, Fig. 6 exemplifies a rather strong dependence of $s(\mathrm{APR})$ on the obliquity - the APR slope decreases rapidly with $\gamma$, and in some cases (not shown here), it may even become negative. It is noted that such negative slopes were found by Kristensen \& Dohrup (1997) and have also recently been detected in TNOs (e.g. Belskaya et al. 2005).

To further investigate the influence of the obliquity, we have (for every irregular body), calculated the APRs for each $\{\beta, \gamma\}$ combination on a $5^{\circ}$ resolution grid $\left(0^{\circ} \leq\{\beta, \gamma\} \leq 90^{\circ}\right)$. Each

\footnotetext{
${ }^{5}$ This is the case at least for monochromatic lightcurves.
} 

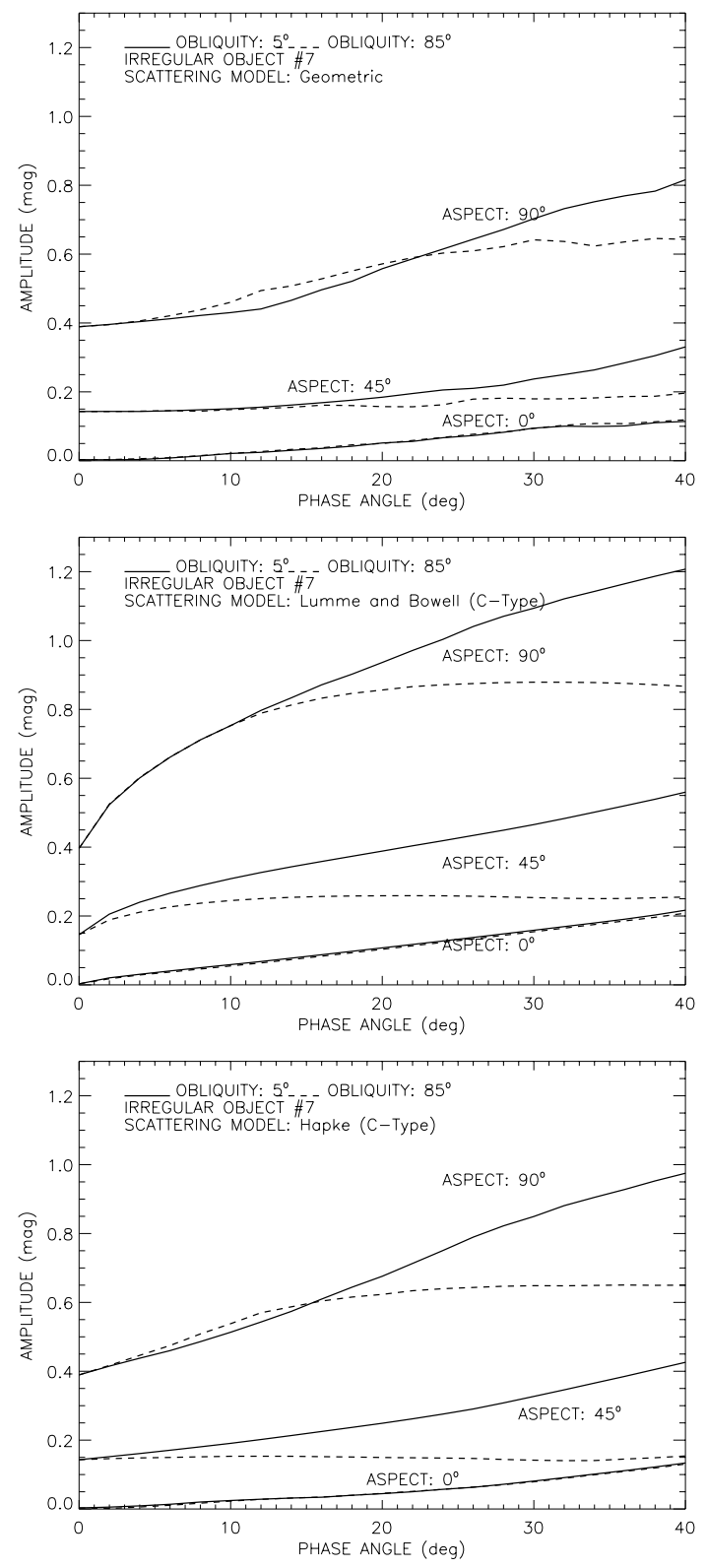

Fig. 6. Lightcurve amplitude versus phase angle (i.e., APRs) for an irregular model object, considering six different combinations of aspect and obliquity angles, as indicated in the figure. The top, middle, and bottom panels show the results obtained for three different scattering laws; geometrical, Lumme-Bowell, and Hapke, respectively.

APR was then normalized by $A\left(0^{\circ}\right)_{\text {LS }}$, obtained by calculating the intersection of the ordinate by a line fitted to the APR in a least-squares (LS) sense ${ }^{6}$. Finally, averaging was made over $\beta$, thereby obtaining a function $M(\alpha)=\left\langle A(\alpha) / A\left(0^{\circ}\right)_{\mathrm{LS}}\right\rangle_{\beta}$ for each considered obliquity angle. It should be noted that perfectly linear APRs would have resulted in $M(\alpha)=1+m \cdot \alpha$, according to Eq. (3).

Figure 7 shows a number of $M(\alpha)$ curves for a particular object for different obliquities (Lumme-Bowell and Hapke scattering for C-type parameters to the left and right, respectively; note that two different irregular bodies are considered). The $M(\alpha)$ curves are quasi-linear for $\alpha \gtrsim 5^{\circ}$, i.e., the slopes

${ }^{6} A\left(0^{\circ}\right)_{\text {LS }}$ was used instead of $A\left(0^{\circ}\right)$ to minimize the influence of nonlinearity in the APRs at small phase angles during the normalization procedure. correspond to $m$ as defined in Eq. (2). As can be seen, the dispersion in $m$ caused by changing the obliquity is significant and covers the whole $0.010 \leq m \leq 0.030 \mathrm{mag} \mathrm{deg}^{-1}$ region, that is, the $m$-values found for C- and S-type asteroids by Zappalà et al. (1990), respectively. It is clearly seen that the slope $m$ decreases with increasing obliquity. In fact, there are examples among the considered bodies (in particular for Hapke scattering), where $m<0 \mathrm{mag} \mathrm{deg}^{-1}$ is found, i.e., the lightcurve amplitude decreases with the phase angle. As previously mentioned, such behavior is also seen in real asteroids and TNOs.

Considering the results for all the studied objects, $m$ is generally $0.020-0.030 \mathrm{mag} \mathrm{deg}^{-1}$ larger for small obliquity angles, than for large ones. This dispersion in $m$ due to obliquity is at least twice the $1-\sigma$ dispersion due to topography discussed in the previous section.

To further illustrate the dependence of the APR on obliquity, and its relative importance versus aspect angle $(\beta)$ and topography, we have fixed a certain obliquity value and calculated $s(\mathrm{APR})$ and $A\left(0^{\circ}\right)_{\mathrm{LS}}$ for all 16 bodies, using a $5^{\circ}$ resolution grid in $0^{\circ} \leq \beta \leq 90^{\circ}$ for each. Using a $0.06 \mathrm{mag}$ binning for $A\left(0^{\circ}\right)_{\mathrm{LS}}, s(\mathrm{APR})$-values originating from different shape $/ \beta$-combinations (but within the same $A\left(0^{\circ}\right)$-bin) were then grouped together and averaged. The result can be seen in Fig. 8 for two different obliquities, $\gamma=0^{\circ}$ (diamonds, $\diamond$ ) and $\gamma=90^{\circ}$ (filled circles, $\bullet$ ). It is important to note that the error bar for each data point shows the $1-\sigma$ dispersion in $s(\mathrm{APR})$ caused by topography and aspect angle.

In Fig. 8, it is clear that the dispersion in $s(\mathrm{APR})$ for a certain $A\left(0^{\circ}\right)$ (i.e., in principle, the dispersion in $m$ ), caused by the topography and aspect angle, are generally smaller than the dispersion introduced by varying the obliquity by $90^{\circ}$. This is evident both for Lumme-Bowell scattering (upper panel) and Hapke scattering (lower panel), at least for the C-type parameters considered here. Furthermore, the linear APRs suggested for C- and S-type asteroids by Zappalà et al. (1990), shown as dashed lines in Fig. 8, agree fairly well with the boundaries of the region in $\left\{A\left(0^{\circ}\right), s(\mathrm{APR})\right\}$ space, created by variations in topography, aspect angle, and obliquity. For Lumme-Bowell scattering, the bodies included in our study produce APRs with $0.011 \leq m \leq 0.033 \mathrm{mag} \mathrm{deg}^{-1}$. The corresponding range for Hapke scattering is $0.002 \leq m \leq 0.023 \mathrm{mag} \mathrm{deg}^{-1}$, i.e., less steep slopes than for Lumme-Bowell scattering. In both cases, the dispersion caused by differences in obliquity angle is therefore about $0.02 \mathrm{mag} \mathrm{deg}^{-1}$.

\subsection{Differences between the Lumme-Bowell and Hapke scattering models}

As has been mentioned previously, the Lumme-Bowell and Hapke scattering theories do not always yield APRs with the same qualitative behavior, even when the same model body, viewing and illumination geometries, and (scattering parameters corresponding to a certain) taxonomic type are considered. Below, we summarize some of the model-dependent differences seen in Figs. 4-8. These differences may not be entirely general, but restricted to the particular cases under study. However, the existence of such differences in at least a number of cases motivates a more detailed study of the problem in the future.

1. The Lumme-Bowell theory predicts a stronger dependence of the APR on single-scattering albedo and cosine asymmetry than does Hapke theory (Fig. 4).

2. Whereas the Hapke model generally yields quasi-linear APRs in the entire considered phase angle interval, the 

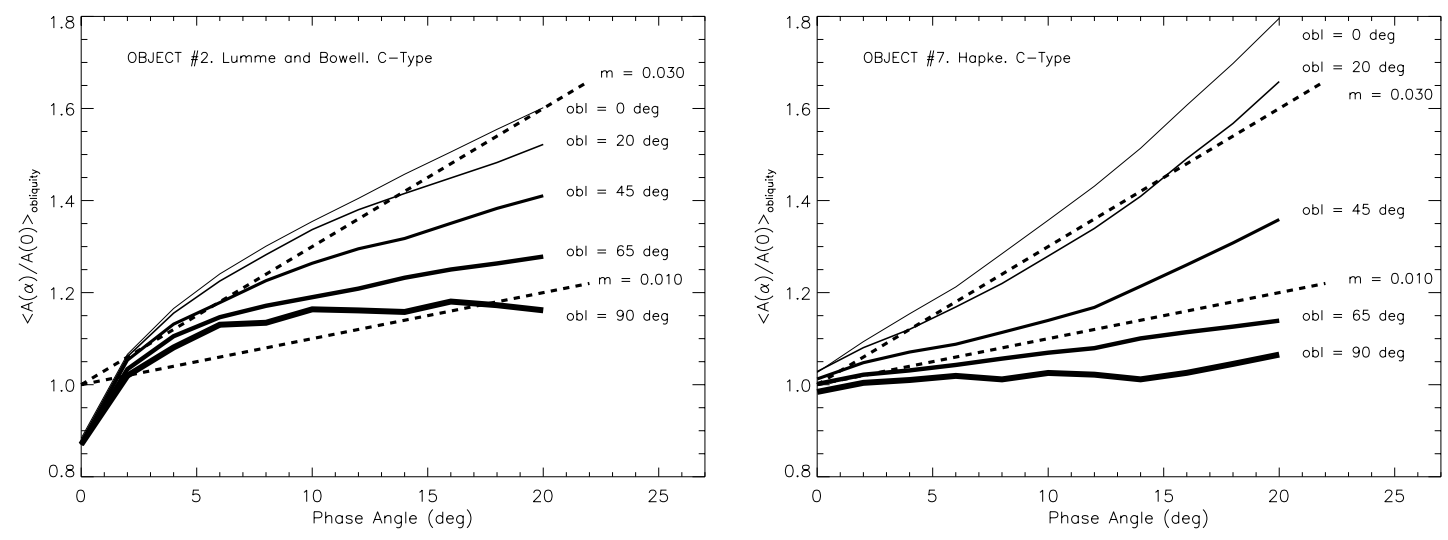

Fig. 7. The function $M(\alpha)=\left\langle A(\alpha) / A\left(0^{\circ}\right)_{\mathrm{LS}}\right\rangle_{\beta}$, plotted for a number of obliquities $\gamma=\left\{0^{\circ}, 20^{\circ}, 45^{\circ}, 65^{\circ}, 90^{\circ}\right\}$. The two dashed lines represent the $m=0.010 \mathrm{mag} \mathrm{deg}^{-1}$ and $m=0.030 \mathrm{mag} \mathrm{deg}^{-1}$ slopes found by Zappalà et al. (1990) for geometric scattering ellipsoids and S-type asteroids, respectively. The left and right panels show the results of using the Lumme-Bowell and Hapke scattering theories, respectively, both assuming C-type parameters (note that two different small objects were considered).

Lumme-Bowell model frequently leads to a strong drop in the lightcurve amplitude close to opposition (Figs. 5-7).

3. The dispersion in $m$ due to obliquity appears to be rather similar for the two scattering models considered, but the Lumme-Bowell theory consistently seems to yield steeper slopes, i.e., higher $m$ values (Figs. 7 and 8).

It is out of the scope of the current paper to explain the reasons for all these differences. We therefore limit our discussion to the second issue, which is probably the most interesting one, since it in principle could be investigated observationally (regardless of the lack of knowledge on $\beta, \gamma$, and the detailed shape of the asteroid). As was already pointed out by Karttunen \& Bowell (1989), and as can be seen in the present work, the Lumme-Bowell law predicts an initially rapid increase in the lightcurve amplitude as the phase angle increases from zero, after which it grows linearly beyond $\alpha \gtrsim 5^{\circ}$. However, the Hapke theory does not display such a behavior, and one might ask about the reason for this qualitative discrepancy.

Tests with a prolate body without topography (elongation $1: 1: 1.55, \beta=90^{\circ}, \gamma=0^{\circ}$ ) reveal that the surface roughness parameter $\rho$ solely is responsible for the initially rapid increase in $A(\alpha)$ seen when Lumme-Bowell scattering is applied, as the phase angle increases from zero. If this parameter is reduced from $\rho=1.17$ (the nominal value for C-type asteroids with the Lumme-Bowell formalism) to $\rho=0.1$, the APR becomes linear and closely resembles the APR obtained with Hapke scattering. Alternatively, if the roughness parameter in the Hapke scattering law is increased from $\theta=20^{\circ}$ (the nominal value for C-type asteroids in that formalism) to $\theta=60^{\circ}$, the APR closely matches the $\rho=1.17$ non-linear Lumme-Bowell APR.

The underlying reason why the amplitude-phase relationships for the Lumme-Bowell and Hapke scattering laws differ for seemingly identical conditions is therefore a different opinion in regards to the degree of surface roughness for C-type asteroids. Phase functions (i.e., asteroid magnitude versus phase) are often used to extract photometric parameters, and thereby physical characteristics, for the asteroids in question. When the Lumme-Bowell theory is applied for phase function inversion, this predicts a rather high degree of surface roughness for C-type asteroids. However, when the Hapke theory is used to invert the same phase functions, the predicted degree of surface roughness is comparably low. The two scattering theories, with their respective parameter sets for C-type asteroids (see Table 2), therefore reproduce the same phase function, but fail to yield the same physical interpretation of the regolith properties. As the two theories also fail to yield the same APR, observations of the nearopposition behavior of the lightcurve amplitude (i.e., if the APR is linear or not) could therefore in principle be used to decide if the asteroid regolith is indeed very rough, or not.

\section{Discussion and summary}

Asteroids are often observed to increase their lightcurve amplitudes with phase angle, and the rate of this increase, $s$ (APR), is often observed to increase with $A\left(0^{\circ}\right)$, the amplitude at zero phase angle. Theoretical studies of macroscopically smooth ellipsoidal bodies with geometrical scattering indicate that the parameter $m=s(\mathrm{APR}) / A\left(0^{\circ}\right)$ is a constant (where the body elongation and aspect angle determines $A\left(0^{\circ}\right)$ ). However, analysis of $m$ for real asteroids indicates a substantial variation among asteroids, and we have considered alternative physical mechanisms behind this dispersion here.

We find that obliquity is the single most important factor determining $m$, and this strong dependence is only evident when irregular bodies with realistic scattering properties are studied instead of geometrically scattering smooth ellipsoids. Topography also contributes to the dispersion in $m$, but to a somewhat lesser extent. Scattering parameters (ultimately reflecting the mineralogy, surface texture, and taxonomic type) do not appear to cause an observable dispersion in $m$.

Remembering that Eqs. (1)-(3) are often used to reduce observed lightcurve amplitudes to a common phase angle (possibly using $m$-values considered suitable for a certain taxonomic type), it is important to reconsider the relevancy of this procedure in the light of the results obtained in the current paper. If the shapes and photometric models used in this study can be considered as representative of reality, the behavior of the obliquity $\gamma$ during the observational window is crucial. If obliquity does not change significantly between observations (which may include several opposition passages), our results suggest that a simple linear relationship between $s(\mathrm{APR})$ and $A\left(0^{\circ}\right)$ (i.e., a constant $m$ ) could be reasonable. However, for large variations in the obliquity, a significant change in the slope $m$ is very likely.

Assuming that spin axes are randomly oriented, the average obliquity for a set of observed asteroids would be $\langle\gamma \sin \gamma\rangle=60^{\circ}$. Our simulations indicate that the average $s(\mathrm{APR}) / A\left(0^{\circ}\right)$ ratio for the objects in this sample would be $m \approx 0.018 \mathrm{mag} \mathrm{deg}^{-1}$ according to the Lumme-Bowell model 

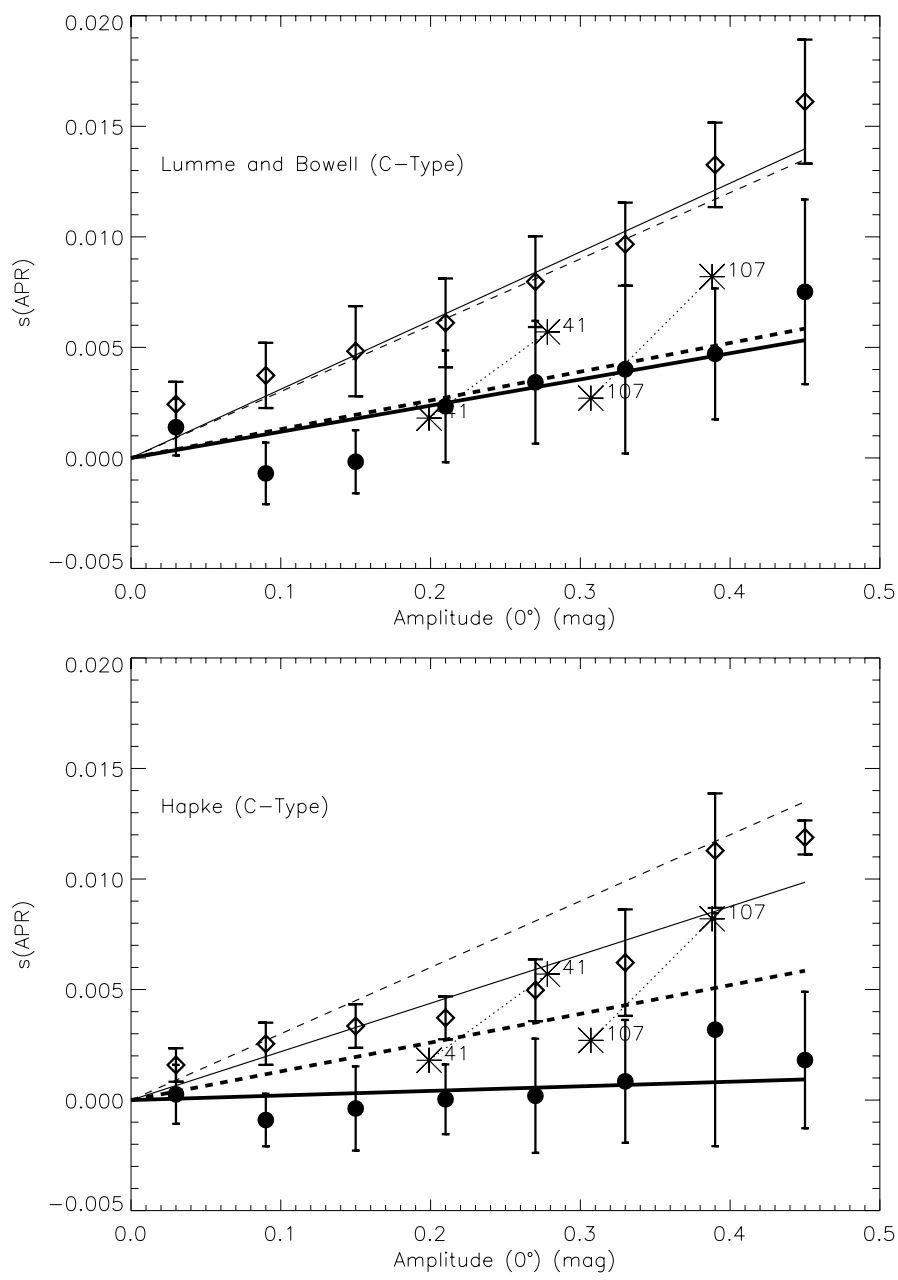

Fig. 8. Averages of $s(\mathrm{APR})$, corresponding to a number of body shape and aspect angle $\left(0^{\circ} \leq \beta \leq 90^{\circ}\right)$ combinations, as a function of $A\left(0^{\circ}\right)$, obtained for two different obliquities, $\gamma=0^{\circ}$ (diamonds, $\diamond$ ) and $\gamma=90^{\circ}$ (filled circles, $\bullet$ ). Vertical error bars represent the $1-\sigma$ dispersion caused by variation in topography and aspect angle (solid lines are leastsquares fits to the data points). The upper and lower panels show results obtained for the Lumme-Bowell and Hapke scattering laws, respectively, both evaluated for C-type scattering parameters. Dashed lines show the linear relationships found by Zappalà et al. (1990) for S-type asteroids (thin line) and for C- and M-type asteroids (thick line). Solid lines show the least-squares fits to the numerical data. Furthermore, asterisks $(*)$ show the particular $\left\{A\left(0^{\circ}\right), s(\mathrm{APR})\right\}$ combinations found by Zappalà et al. (1990) for Asteroids 107 Camilla and 41 Daphne (two oppositions considered for each asteroid).

and $m \approx 0.010 \mathrm{mag} \mathrm{deg}^{-1}$ according to the Hapke model. These numbers are similar to the average value found by Kristensen \& Dohrup (1997), i.e., $m=0.0120 \pm 0.0046 \mathrm{mag} \mathrm{deg}^{-1}$. The dispersion of $m$-values around this mean value (see Figs. 1 and 2), including the presence of data points with $m<0 \mathrm{mag} \mathrm{deg}^{-1}$, is most likely explained entirely by individual variations in obliquity and shape topography, according to our results.

Focusing on the lightcurve analysis by Zappalà et al. (1990), we may consider the asteroids in their sample for which two or more estimates of $s(\mathrm{APR})$ exist (i.e., the amplitude has been measured during at least two oppositions, comprising different aspect angles, and presumably different mean obliquity angles). It is found that some of the asteroids (in particular 16 Psyche, 41 Daphne, and 107 Camilla), can hardly be characterized by a particular (constant) $m$-value. In Fig. 8, we have superimposed the $\left\{A\left(0^{\circ}\right), s(\mathrm{APR})\right\}$ data for two of these asteroids (41 Daphne

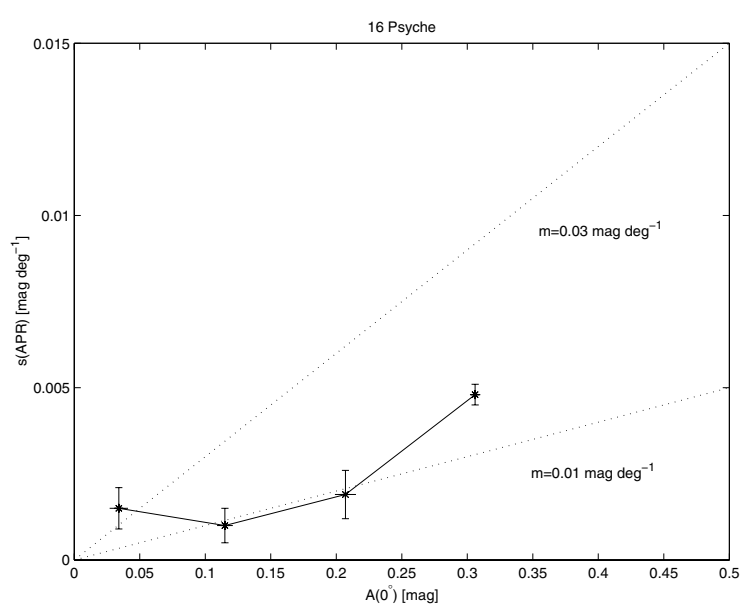

Fig. 9. Amplitude-phase relationship slopes $s$ (APR) versus zero-phase lightcurve amplitudes $A\left(0^{\circ}\right)$ obtained for 16 Psyche at four different oppositions. The measured data does not conform with a line passing through the origin, which probably means that the mean obliquity angles were different during the oppositions.

and 107 Camilla). The data points (marked with asterisks, *, and accompanied by the asteroid number), show that $m=$ $s(\mathrm{APR}) / A\left(0^{\circ}\right)$ has varied among oppositions (the data points do not lie on a line passing through the origin).

Yet another example can be seen in Fig. 9, for asteroid 16 Psyque. The data points clearly do not follow a linear relation, i.e., a single $m$ can hardly be specified. Our interpretation is that the mean obliquity probably has varied between oppositions. Possibly, the two intermediate data points for 16 Psyche were obtained for the same (large) mean obliquity, since they closely conform with the $m=0.01 \mathrm{mag} \mathrm{deg}^{-1}$ line. However, the other two data points were probably obtained during oppositions when the mean obliquity was small to intermediate. Nonetheless, at this point, this interpretation is certainly speculative, and unfortunately, lack of observational material does not allow us to draw any definitive conclusions.

To summarize, we arrive at the following conclusions.

1. For complex scattering and shape models, obliquity is found to have a strong effect on the APR. The slope $m$ is found to decrease with obliquity (a $90^{\circ}$ change in $\gamma$ leads to a $\sim 0.02 \mathrm{mag} \mathrm{deg}^{-1}$ change in $\mathrm{m}$ ). This dispersion is sufficient to explain differences in $m$ among observed asteroids. In our simulations, it is possible to find cases where $m<0 \mathrm{mag} \mathrm{deg}^{-1}$ (i.e., the lightcurve amplitude decreases with the phase angle), in particular for intermediate aspect angles. Such APRs can also be found among real asteroids and TNOs.

2. Our simulations indicate that the average obliquity $\gamma=60^{\circ}$, expected for a population with fully randomized rotational axes, leads to an average $s(\mathrm{APR}) / A\left(0^{\circ}\right)$ ratio of $0.010 \leq m \leq$ $0.018 \mathrm{mag} \mathrm{deg}^{-1}$. This is consistent with the empirical results obtained by Kristensen \& Dohrup (1997).

3. Topographic features on a body introduce shadowing effects, which have a comparatively strong influence on the amplitude-phase relationship. The $1-\sigma$ dispersion in $m$ caused by topography is about half as large as that obtained for obliquity variations, $\sim 0.01 \mathrm{mag} \mathrm{deg}^{-1}$.

4. Variation of the parameters entering into the Lumme-Bowell and Hapke models has a negligible effect on the zero-phase amplitude and the APR slope. We thereby confirm the results obtained previously by Karttunen \& Bowell (1989) and 
extend this statement to include Hapke theory. We therefore do not find support for the suggestion by Zappalà et al. (1990) that S-type asteroids have systematically larger $m$-values than $\mathrm{C}$ - and M-type asteroids.

5. Differences are seen in APRs calculated with the LummeBowell theory with respect to the Hapke theory, in spite of the fact that identical body shapes, illumination and viewing geometries, as well as taxonomic types are considered (here, C-type). In particular, the behavior of the APR close to the zero phase is different, being strongly non-linear for Lumme-Bowell scattering and nearly linear for Hapke scattering. The reason is that the average Lumme-Bowell parameter set for C-type asteroids indicates that these bodies have a substantial surface roughness, while a similar average Hapke parameter set indicates the contrary. It is noted that the two models (with their respective parameter sets) reproduce the same C-type phase functions, but that the difference in surface roughness becomes evident when the amplitude-phase relationship is considered. This indicates that APRs potentially contain additional important information, which could be utilized during reflectance inversion.

It is worthwhile to note that both complex scattering models may show similar amplitude-phase behavior for particular values of their scattering parameters, as shown in Sect. 4.4. In particular, a roughness parameter of about $\theta=60^{\circ}$ in the Hapke theory will yield a similar non-linear APR, as seen in our Lumme-Bowell calculations. On the contrary, a roughness of about $\rho=0.1$ in the Lumme-Bowell theory will produce a similar linear APR to that of the Hapke theory. Nevertheless, such values for the corresponding parameters are not found for observed objects.

6. Given the uncertainty in $m$, introduced primarily by the obliquity angle (which generally is unknown), we recommend caution when attempting to reduce asteroid lightcurve amplitudes to a common phase angle. For asteroids with a known obliquity, we recommend using the relation $m \approx$ $0.0280-0.0003 \gamma[\mathrm{deg}]$ during the reduction.

Acknowledgements. P.J.G. acknowledges financial support from the Spanish Ministerio de Educación y Ciencia (contract "Juan de la Cierva"). The research carried out has been partially supported by the Spanish Ministerio de Ciencia y Tecnología under contract ESP2003-00357. Davidsson acknowledges financial support from SNSB contract 133/04:2.

\section{References}

Barucci, M. A., \& Fulchignoni, M. 1982, The Moon and the Planets, 27, 47 Belskaya, I., Ortiz, J. L., Rousselot, P., et al. 2005, Icarus, submitted

Buratti, B. J., Hicks, M. D., Soderblom, L. A., et al. 2004, Icarus, 167, 16 Gehrels, T. 1956, ApJ, 123, 331

Gutiérrez, P. J., Ortiz, J. L., Rodrigo, R., \& López-Moreno, J. J. 2000, A\&A, 355,809

Hapke, B. W. 1981, J. Geophys. Res., 86, 3039

Hapke, B. W. 1984, Icarus, 59, 41

Helfenstein, P., \& Ververka, J. 1989, in Asteroids II, ed. R. Binzel, T. Gehrels, \& M. S. Matthews, 557

Karttunen, H. 1989, A\&A, 208, 314

Karttunen, H., \& Bowell, E. 1989, A\&A, 208, 320

Kristensen, L. K., \& Dohrup, P. 1997, Planet. Space Sci., 45, 751

Lumme, K., \& Bowell, E. 1981a, AJ, 86, 1694

Lumme, K., \& Bowell, E. 1981b, AJ, 86, 1705

Lumme, K., Bowell, E., \& Zellner, B. 1980, Lunar and Planetary Science VI, 637

Magnusson, P. 1986, Icarus, 68, 1

Muinonen, K. 1996, EM\&P., 72, 229

Muinonen, K. 1998, A\&A, 332, 1087

Muinonen, K., \& Lagerros, J. S. V. 1998, A\&A, 333, 753

Peltoniemi, J. I., Lumme, K., Muinonen, K., \& Irvine, W. M. 1989, Appl. Opt., 28,4088

Pospieszalska-Surdej, A., \& Surdej, J. 1985, A\&A, 149, 186

Russell, H. 1906, ApJ, 24, 1

Zappalà, V., Cellino, A., Barucci, M. A., Fulchignoni, M., \& Lupishko, D. F. 1990, A\&A, 231, 548 